OPEN ACCESS

Edited by:

Yi Yang,

Jilin University, China

Reviewed by:

Fang Deng,

Jilin University, China

Junlei Chang,

Chinese Academy of Sciences, China

*Correspondence:

Wei Zhang

ttyyzw@163.com

Specialty section: This article was submitted to Mood and Anxiety Disorders, a section of the journal

Frontiers in Psychiatry

Received: 04 June 2018 Accepted: 07 December 2018 Published: 18 January 2019

Citation:

Lian T-H, Guo P, Zuo L-J, Hu Y, Yu S-Y, Liu L, Jin Z, Yu Q-J, Wang R-D, Li L-X

Piao Y-S and Zhang W (2019) An Investigation on the Clinical Features and Neurochemical Changes in Parkinson's Disease With Depression.

Front. Psychiatry 9:723 doi: 10.3389/fpsyt.2018.00723

\section{An Investigation on the Clinical Features and Neurochemical Changes in Parkinson's Disease With Depression}

\author{
Teng-Hong Lian ${ }^{1}$, Peng Guo ${ }^{1}$, Li-Jun Zuo ${ }^{1}$, Yang Hu ${ }^{1}$, Shu-Yang Yu ${ }^{1}$, Li Liu ${ }^{2}$, Zhao Jin ${ }^{1}$, \\ Qiu-Jin Yu ${ }^{1}$, Rui-Dan Wang ${ }^{1}$, Li-Xia Li ${ }^{2}$, Ying-Shan Piao ${ }^{3}$ and Wei Zhang ${ }^{4,5,6,7,8 *}$ \\ ${ }^{1}$ Department of Neurology, Beijing Tiantan Hospital, Capital Medical University, Beijing, China, ${ }^{2}$ Department of Internal \\ Medicine, Beijing Tiantan Hospital, Capital Medical University, Beijing, China, ${ }^{3}$ Center for Movement Disorder, Department of \\ Neurology, Beijing Tiantan Hospital, Capital Medical University, Beijing, China, ${ }^{4}$ Center for Cognitive Neurology, Department \\ of Neurology, Beijing Tiantan Hospital, Capital Medical University, Beijing, China, ${ }^{5}$ China National Clinical Research Center for \\ Neurological Diseases, Beijing, China, ${ }^{6}$ Key Laboratory for Neurodegenerative Disorders of the Ministry of Education, Capital \\ Medical University, Beijing, China, ' Center of Parkinson's Disease, Beijing Institute for Brain Disorders, Beijing, China, \\ ${ }^{8}$ Beijing Key Laboratory on Parkinson's Disease, Beijing, China
}

Objective: To investigate the clinical features and neurochemical changes in Parkinson's disease with depression (PD-D).

Methods: A total of 478 PD patients were divided into PD-D and PD patients without depression (PD-ND) groups according to the 24-item Hamilton Depression Rating Scale (HAMD) score. Demographic variables, motor and non-motor symptoms and activities of daily living were evaluated. The independent influencing factors of PD-D were investigated via binary logistic regression analysis. The levels of neurotransmitters in cerebrospinal fluid (CSF) were measured and their correlations with HAMD score were analyzed.

Results: The proportion of PD-D was 59.0\%, of which 76.95, 20.92, and $2.13 \%$ had mild, moderate, and severe depression, respectively. Anxiety/somatization was the most prevalent sub-factor of HAMD in PD-D. The scores of UPDRS III, postural instability/gait difficulty (PIGD) type and the scores of 14-item Hamilton Anxiety Scale (HAMA) and 14item Chalder Fatigue Scale (FS) were independently associated with PD-D. The levels of dopamine (DA) and 5-hydroxytryptamine (5-HT) were all significantly reduced in PD-D group compared with those in PD-ND group. HAMD scores were negatively correlated with the DA levels in CSF.

Conclusions : PD patients have a high proportion of depression, mainly of mild and moderate levels. The profile of depression in PD population is subtly different from that of the general population. Motor symptoms, PIGD type, anxiety and fatigue are the significant influencing factors of PD-D. Compared to 5-HT, DA may play a more important role in $\mathrm{PD}-\mathrm{D}$.

Keywords: depression, Parkinson's disease, risk factor, dopamine, serotonin 


\section{INTRODUCTION}

Parkinson's disease (PD) is a common neurodegenerative disorder with both the characteristic motor symptoms and a variety of non-motor symptoms. Depression, one of the most common non-motor symptoms of PD (1), used to be regarded as a psychological reaction to $\mathrm{PD}$, and thus frequently be underestimated and undertreated. The failure to detect PD with depression (PD-D) and offer timely treatment would lead to worse outcomes for patients and caregivers (2).

Clinically, studies on PD-D have yielded conflicting results. In terms of demographic information, for example, some investigators showed that the frequency of PD-D increased with age (3), while others claimed that younger patients were more susceptible to PD-D (4). Some studies identified that being female and having a longer disease duration were the risk factors for PDD (5), but some did not (6). From the aspect of symptoms, worse motor symptoms might be associated with PD-D (7). However, there were few studies that investigated the relationship between clinical type of PD and PD-D. For non-motor symptoms, most studies agreed that anxiety was the independent influencing factor (8), but other non-motor symptoms, for instance, fatigue, cognitive decline and sleep disturbances, had considerably varied reports $(4,9)$. Therefore, there is a lack of consensus on the influencing factors of PD-D. Studies consisting of large samples are necessary to explore both general and specific factors related to PD-D. In addition, there are few studies investigating the sub-factors of the 24-item Hamilton Depression Scale (HAMD) in PD-D.

The underlying mechanisms of PD-D have not been adequately clarified. Currently, PD-D is known to be of biological cause, and may be affected by multiple factors including genetic predisposition, biochemical disturbances, and psychological events $(7,10)$. The altered levels of neurotransmitters may serve as the biochemical basis of depression. 5-hydroxytryptamine (5-HT), dopamine (DA), and norepinephrine $(\mathrm{NE})$, which were altered in the pathological process of $\mathrm{PD}$, were known to play important roles in primary depression (11). Therefore, the pathophysiology of PD-D might also be related to the changes in the serotonergic, dopaminergic and noradrenergic systems (12). However, the changes in the above neurotransmitters for PD-D patients may not be the same as those in patients with idiopathic depression, which major biochemical compromise is the serotonergic system. To our best knowledge, there are limited studies on the neurotransmitters levels in cerebrospinal fluid (CSF) from PD-D patients.

In this study, PD-D was assessed using HAMD (13); motor symptoms and other non-motor symptoms were evaluated by a series of rating scales; the levels of 5HT, DA, and NE in the CSF were measured by high performance liquid chromatography (HPLC), and their correlations with HAMD scores were analyzed. The objectives of this study were to investigate the clinical features, influencing factors and neurochemical mechanisms of PD-D.

\section{METHODS}

\section{Ethics Statement}

This study met the guidelines of Helsinki Declaration on ethical principles for medical research involving human subjects, and the protocol was approved by the ethical review board of Beijing Tiantan Hospital. All participants signed written informed consents before they were recruited in the study.

\section{Participants}

Total 500 PD patients were consecutively recruited from the Department of Neurology and Geriatrics of the Beijing Tiantan Hospital from November 2014 to November 2017. Patients were diagnosed with idiopathic PD based on the criteria of the Parkinson's UK Brain Bank (14).

Exclusion criteria included severe systemic diseases, such as heart failure, pulmonary disease, gastrointestinal disorders, anemia, infectious disease and chronic inflammatory disease, deep brain stimulation, and conditions that might interfere with the reliable completion of clinical assessments.

Demographic variables including sex, age, age of onset, disease duration, side of onset, education level and anti-parkinson therapy, including the types of drugs, levodopa equivalent daily dose (LEDD) and the durantion of taking medicines, etc. LEDD was calculated as previously proposed (15).

\section{Clinical Assessment For PD Patients Depression}

PD-D was diagnosed according to the diagnostic criteria for depression in PD (16). Patients with depression resulted from systemic diseases, organic mental disorders, psychoactive substances or non-addictive substances and patients on antidepressants were excluded from analyses.

Twenty-two patients were excluded due to ineligibility. A final total of 478 PD patients were included in the research. Patients with HAMD score of $\geq 8$ points and fulfilled the above diagnostic criteria were assigned to the PD-D group. Within the PD-D group, patients with HAMD scores of $8-19$ points, $20-34$ points and $\geq 35$ points were categorized as having mild, moderate and severe depression, respectively. PD patients with HAMD scores of $<8$ points were assigned to the group of $\mathrm{PD}$ patients without depression (PDND).

The 24 items of HAMD can be grouped into the following 7 factors (5): (1) anxiety/somatization (6 items: psychic anxiety, somatic anxiety, gastrointestinal symptoms, hypochondriasis, insight, and general symptoms); (2) weight loss (1 item); (3) cognitive disturbances (6 items: self-guilt, suicide, agitation, depersonalization and derealization, paranoid, and obsessivecompulsive symptom); (4) circadian fluctuations (1 item); (5) retardation symptoms (4 items: depression, work and interests, retardation, and sexual symptoms); (6) sleep disturbances (3 items: difficulty falling asleep, superficial sleep and early awakening); (7) hopelessness symptoms (3 items: helplessness, hopelessness and worthlessness). 
Motor Function, Non-motor Symptoms, and Activities of Daily Living

The severity of PD was evaluated according to the Hoehn and $\mathrm{Yahr}(\mathrm{H}-\mathrm{Y})$ stage. The motor symptoms were assessed using the Unified Parkinson's Disease Rating Scale (UPDRS) III. PD patients were divided into three phenotypes according to the clinical phenotype classification by Jankovic (17): tremordominant (TD) subtype, postural instability and gait difficulty (PIGD) subtype and mixed subtype. Items 16,20 , and 21 of the UPDRS were for tremor symptoms; items $13,14,15,29$, and 30 of UPDRS were for PIGD symptoms. The phenotype was defined by the ratio of the mean tremor score to PIGD score. TD subtype was defined as the ratio $\geq 1.5$, PIGD subtype as the ratio $\leq 1.0$ and mixed subtype as the ratio between 1.0 and 1.5 .

A variety of non-motor symptoms were assessed using the following scales: the Montreal Cognitive Assessment Scale (MoCA) (18) for cognitive impairment, the 14-item Hamilton Anxiety Scale (HAMA) (19) for anxiety, the 14-item Chalder Fatigue Scale (FS) (20) for fatigue, the Rapid Eye Movement Sleep Behavior Disorder (RBD) Screening Questionnaire (RBDSQ) (21) for RBD, the Scale for Outcomes in PD for Autonomic Symptoms (SCOPA-AUT) (22) for autonomic dysfunction, and the Restless Legs Syndrome (RLS) Severity Rating Scale (RLSRS) (23) for RLS.

Activities of daily living (ADL) were evaluated by ADL Scale.

\section{Measurements of Neurotransmitters in CSF From PD Patients}

Anti-parkinsonian drugs were withdrawn for $12-14 \mathrm{~h}$ if the patients' condition allowed and longer time was considered unethical by our ethical committee. For medical washout period should be three times of the $t 1 / 2$ of the medicine, the subjects taking drugs with long $\mathrm{t} 1 / 2$, including controlled release Sinemet (Sinement CR), Pramipexole and controlled release Piribedil (Piribedil CR) were excluded in the analysis of neurotransmitters. Under fasting condition, $5 \mathrm{ml} \mathrm{CSF}$ was taken in a polypropylene tube through lumbar puncture, between 7 and 9 a.m. CSF samples were centrifuged immediately at $3,000 \mathrm{rpm}$ at $4^{\circ} \mathrm{C}$. Then, approximately $0.5 \mathrm{ml}$ volume of CSF was aliquoted into separate Nunc cryotubes and kept frozen at $-80^{\circ} \mathrm{C}$ until usage in the assays.

The levels of neurotransmitters, including DA, 5-HT, and NE in CSF from PD patients were measured by HPLC (24). LC-MS-MS 6410 chromatograph and Phenomenex $150 * 2 \mathrm{~mm}$ and $150^{*} 3 \mathrm{~mm}$ chromatographic columns were from the Agilent Company (California, USA), and the standard sample was from Sigma Company (California, USA). In total PD patients, 89 patients agreed lumbar puncture and after excluding patients used drugs with long T1/2, 76 patients' neurotransmitters in CSF were analyzed.

\section{Statistical Analyses}

Statistical analyses were performed using SPSS Statistics 20.0 (IBM Corporation, $220 \mathrm{New}$ York, USA). $P$-value of less than an alpha level of 0.05 was defined as statistically significant.

Demographic information, motor and non-motor symptoms and ADL scores between PD-D and PD-ND group were compared. Continuous variables, if normally distributed, were presented as means \pm SDs and the 2 groups were compared using 2-tailed $t$-test; if not normally distributed, the data were presented as median (quartile) and compared using nonparametric test. Discrete variables were compared using Chisquare test.

Binary logistic regression analysis was used to investigate the independent influencing factors of PD-D. The covariates with statistical differences in single-factor analysis were divided into a multivariate model; Backward elimination was applied to remove non-significant variables. Influencing factors were presented as odd ratios (OR) with $95 \%$ confidence intervals (CI).

Three kinds of neurotransmitters levels of CSF between PD-D and PD-ND groups were compared. Bonferroni correction was made and $\mathrm{P}$ was reduced to $0.017(0.05 / \mathrm{n}=0.05 / 3 \approx 0.017)$. Pearson correlation analyses were conducted between HAMD scores and neurotransmitters levels in CSF in the PD group. Binary logistic regression analysis was used. The covariates contained the neurotransmitters which had statistical differences in single-factor analysis and the independent influencing factors of PD-D in the above mentioned binary logistic regression analysis; Backward elimination was applied to remove nonsignificant variables.

\section{RESULTS}

\section{Frequency of Depression of PD}

Among 478 PD patients, 282 (59.00\%) were diagnosed with PDD: of which, $76.95 \%$ (217/282 cases) had mild depression, $20.92 \%$ (59/282 cases) had moderate depression, and 2.13\% (6/282 cases) had severe depression. The remaining 196 cases $(41.00 \%)$ were in the PD-ND group.

\section{Assessment of Each Sub-factor of HAMD for PD-D and PD-ND Groups}

The assessment of each sub-factor of HAMD was shown in Table 1. Anxiety/somatization was the most common subfactor reported in PD-D group at $99.30 \%$, followed by retardation symptoms $(97.51 \%)$ and hopelessness symptoms (89.71\%). Anxiety/somatization also occurred in the largest frequency of PD-ND patients, but compared with those of PD-D, the frequency of each sub-factor of HAMD was much lower.

\section{Demographic Variables, Motor Function, Non-motor Symptoms, and ADL of PD-D and PD-ND Groups}

Demographic variables, motor function, non-motor symptoms and ADL scores were compared between PD-D and PD-ND groups in Table 2.

Compared with the PD-ND group, the PD-D group showed a significantly earlier age of onset, remarkably longer disease duration, and significantly lower education level. There was no significant difference in the other demographic variables, 
TABLE 1 | Assessment of each sub-factor of HAMD for PD-D and PD-ND groups.

\begin{tabular}{|c|c|c|c|c|c|c|}
\hline & \multicolumn{2}{|c|}{ Frequency $(n, \%)$} & \multicolumn{2}{|c|}{ Score (mean $\pm S D$ ) } & \multicolumn{2}{|c|}{ Range of the score (min-max) } \\
\hline & PD-ND & PD-D & PD-ND & PD-D & PD-ND & PD-D \\
\hline Anxiety/somatization & 107 (54.60\%) & $280(99.30 \%)$ & $1.08 \pm 1.21$ & $4.64 \pm 2.48$ & $0-5$ & $0-13$ \\
\hline Retardation symptoms & $93(47.40 \%)$ & $275(97.51 \%)$ & $0.69 \pm 0.88$ & $3.53 \pm 1.86$ & $0-4$ & $0-12$ \\
\hline Hopelessness symptoms & 89 (45.40\%) & $253(89.71 \%)$ & $0.69 \pm 0.90$ & $2.76 \pm 1.89$ & $0-4$ & $0-10$ \\
\hline Sleep disturbances & $60(30.60 \%)$ & $211(74.82 \%)$ & $0.51 \pm 0.96$ & $2.30 \pm 1.84$ & $0-5$ & $0-6$ \\
\hline Cognitive disturbances & $28(14.30 \%)$ & $200(70.92 \%)$ & $0.14 \pm 0.35$ & $1.79 \pm 1.95$ & $0-1$ & $0-10$ \\
\hline Weight loss & 28 (9.20\%) & $100(35.46 \%)$ & $0.12 \pm 0.41$ & $0.49 \pm 0.73$ & $0-3$ & $0-3$ \\
\hline Circadian fluctuations & $12(6.10 \%)$ & $97(34.40 \%)$ & $0.10 \pm 0.41$ & $0.63 \pm 0.97$ & $0-4$ & $0-4$ \\
\hline
\end{tabular}

including sex, age, side of onset and the condition usage of the anti-Parkinson drugs between the two groups.

The PD-D group had a significantly advanced H-Y stage, significantly increased UPDRS III score and higher proportion of the PIGD subtype.

The PD-D group scored significantly lower MoCA score and higher scores of HAMA, FS, RBDSQ, SCOPA-AUT, and RLSRS scales when compared with the PD-ND group, demonstrating that the PD-D group had more severe cognitive impairment, anxiety, fatigue, RBD, autonomic dysfunction, and RLS.

Compared with the PD-ND group, the score of ADL scale in the PD-D group was significantly decreased, suggesting that the PD-D group had significantly poor ADL.

\section{Factors Associated With PD-D}

Binary logistic regression analysis was performed using the above variables that found statistical differences between PD-D and PDND groups. The results showed that the scores of UPDRS III, PIGD type, and the scores of HAMA and FS were independently associated with PD-D (Table 3).

\section{Levels of Neurotransmitters in CSF From PD-D and PD-ND Groups}

The levels of DA, 5-HT, and NE in CSF from the PD-ND and PD-D groups were compared (Table 4). In the PD-D group, the levels of DA and 5-HT were all reduced compared with those in the PD-ND group. However, only DA level showed a significant difference after Bonferroni correction.

The correlational analyses between HAMD scores and neurotransmitters levels in CSF from PD patients were further conducted (Table 5). It was found that the HAMD scores had a negative correlation with DA level in CSF $(r=-0.278, P<0.05)$. However, no significant relationship between HAMD scores and the levels of 5-HT and NE in CSF was detected.

Binary logistic regression analysis was performed to investigate the relationship of $\mathrm{PD}-\mathrm{D}$ and $\mathrm{DA}$ levels in CSF. The covariates contained the levels of DA in CSF and the scores of UPDRS III, PIGD type and the scores of HAMA and FS (Table 6). The results showed that the DA levels significantly negatively correlated with PD-D.

\section{DISCUSSION}

Though PD was previously characterized by the classical motor symptoms, recent studies have suggested that non-motor symptoms play significant roles in the deterioration of the quality of life for PD patients $(25,26)$. As one of the mood disorders, depression is often covered by motor symptoms and fails to present as the chief complaint. It is important to diagnose PD$\mathrm{D}$, because it is one of the main determinants of quality of life for PD patients and lack of PD-D diagnosis results in heavy burden to the families of patients $(27,28)$.

The prevalence of PD-D differs greatly across studies, ranging from 2.7 to $90 \%$, and around 35\% of PD patients presented significant depressive symptoms clinically (29), which was much higher than that in general population (30). The variation might be resulted from different diagnostic criteria, rating scales, sample sizes, and study population (31). In the current study, a large sample containing 478 Chinese PD patients was established, of which, $59 \%$ of the population suffered from depression. Analysis of the severity of depression in PD patients indicated that mild, moderate, and severe depression accounted for 76.95, 20.92, and $2.13 \%$, respectively, implying that $\mathrm{PD}-\mathrm{D}$ was featured by the mild and moderate depression (9). Clinical trials have shown that mild depressive symptoms are a variable process and may result in remission or take a turn for more severe and persistent symptoms over time (32).

Previous studies rarely investigated the 7 sub-factors of HAMD-24 items in detail. In this investigation, the results showed that PD-D patients had the highest frequency of anxiety/somatization, which was followed by the symptoms of retardation and hopelessness. The cognitive disturbances, which contains suicide, had the fifth frequency. This suggested that the profile of depression in PD population was subtly different from that of the general population which showed a high rate of suicide (33).

In this study, the binary logistic regression analysis showed that the UPDRS III score, PIGD type, the scores of HAMA and FS were the significant influencing factors of PD-D. Motor symptoms were significantly evidently different between PD$\mathrm{D}$ and PD-ND groups. Some earlier studies failed to find a significant relationship between motor symptoms and PD-D $(9,34)$; the inconsistency may be caused by patients' stronger perception of depression than actual disability in "on" phase. 
TABLE 2 | Demographic variables, motor function, non-motor symptoms, and ADL of PD-D and PD-ND groups.

\begin{tabular}{|c|c|c|c|}
\hline & PD-ND group (196 cases) & PD-D group (282 cases) & $\boldsymbol{P}$ \\
\hline \multicolumn{4}{|l|}{ DEMOGRAPHIC INFORMATION } \\
\hline Female ( $n, \%)$ & 87 (44.39\%) & $150(53.19 \%)$ & 0.058 \\
\hline Age (year, $\bar{X} \pm s)$ & $63.00(56.00-70.00)$ & $61.50(55.75-69.00)$ & 0.278 \\
\hline Age of onset (year, $\bar{X} \pm s$ ) & $59.87 \pm 11.16$ & $57.51 \pm 10.89$ & $0.024^{*}$ \\
\hline Disease duration [year, Median (Q1-Q3)] & $2.00(1.00-4.00)$ & $3.00(1.00-5.00)$ & $<0.001^{\star \star}$ \\
\hline Low education level (<9 years) $(n, \%)$ & $93(47.45 \%)$ & $176(62.41 \%)$ & $<0.001^{\star \star}$ \\
\hline Left side of onset (n, \%) & $82(41.84 \%)$ & $132(46.81 \%)$ & 0.282 \\
\hline \multicolumn{4}{|l|}{ ANTI-PARKINSON DRUG } \\
\hline LEDD [mg, M (Q1-Q3)] & $0.75(0,6.90)$ & $1.16(0,3.00)$ & 0.341 \\
\hline \multicolumn{4}{|l|}{ Kinds of Anti-parkinson drug } \\
\hline Madopar (n, \%) & 85 (43.37\%) & $144(51.06 \%)$ & 0.098 \\
\hline Sinemet CR $(n, \%)$ & $12(6.12 \%)$ & $20(7.09 \%)$ & 0.677 \\
\hline Entacapone (n, \%) & $7(3.57 \%)$ & $19(6.74 \%)$ & 0.133 \\
\hline Pramipexole (n, \%) & 45 (22.96\%) & $48(17.02 \%)$ & 0.107 \\
\hline Piribedil CR (n, \%) & 36 (18.37\%) & 49 (17.38\%) & 0.780 \\
\hline Selegiline $(n, \%)$ & $4(2.04 \%)$ & $4(1.42 \%)$ & 0.722 \\
\hline Antane $(n, \%)$ & 9 (4.59\%) & $23(8.16 \%)$ & 0.125 \\
\hline \multicolumn{4}{|l|}{ Duration of anti-PD therapy } \\
\hline$\leq 1$ year & $61(31.12 \%)$ & $82(29.08 \%)$ & 0.059 \\
\hline$>1-\leq 3$ years & 84 (42.86\%) & 96 (34.04\%) & \\
\hline$>3-\leq 7$ years & 35 (17.86\%) & $64(22.70 \%)$ & \\
\hline >7years & $16(8.16 \%)$ & $40(14.18 \%)$ & \\
\hline \multicolumn{4}{|l|}{ MOTOR FUNCTION } \\
\hline \multicolumn{4}{|l|}{ H-Y stage $(n, \%)$} \\
\hline Early stage (stage 1-2.5) & 179 (91.33\%) & $224(79.43 \%)$ & $<0.001^{\text {** }}$ \\
\hline Advanced score (stage 3-5) & $17(8.67 \%)$ & $58(20.57 \%)$ & \\
\hline Total UPDRS III [point, Median (Q1-Q3)] & $18.00(12.00-27.00)$ & $28.00(19.00-40.00)$ & $<0.001^{\text {** }}$ \\
\hline Tremor & $3.00(2.00-6.00)$ & $5.00(2.00-8.00)$ & \\
\hline Rigidity & $3.00(1.00-5.00)$ & $5.00(2.00-9.00)$ & \\
\hline Bradykinesia & $7.00(5.00-11.00)$ & $11.00(7.00-16.00)$ & \\
\hline Postural instability/gait difficulty & $3.00(2.00-4.00)$ & $4.00(3.00-6.00)$ & \\
\hline \multicolumn{4}{|l|}{ CLINICAL TYPE (n, \%) } \\
\hline TD subtype & $67(35.40)$ & $6(2.70)$ & $<0.001^{\star \star}$ \\
\hline PIGD subtype & $106(52.40)$ & $276(97.30)$ & \\
\hline Mixed subtype & $25(12.20)$ & $0(0.00)$ & \\
\hline \multicolumn{4}{|l|}{ NON-MOTOR SYMPTOMS } \\
\hline MoCA [point, Median (Q1-Q3)] & 23.00 (19.00-27.00) & $21.00(16.00-25.00)$ & $<0.001^{\star *}$ \\
\hline HAMA [point, Median (Q1-Q3)] & $3.00(1.00-5.00)$ & $12.00(8.00-17.75)$ & $<0.001^{\star \star}$ \\
\hline FS [point, Median (Q1-Q3)] & $7.00(4.00-10.00)$ & $10.00(8.00-12.00)$ & $<0.001^{\star \star}$ \\
\hline RBDSQ [points, Median (Q1-Q3)] & $1.00(0.00-4.00)$ & $3.00(1.00-7.00)$ & $<0.001^{\star \star}$ \\
\hline SCOPA-AUT [points, Median (Q1-Q3)] & $33.00(29.00-37.00)$ & $36.00(32.00-43.00)$ & $<0.001^{* *}$ \\
\hline RLSRS [point, Median (Q1-Q3)] & $0.00(0.00-6.00)$ & $0.00(0.00-18.00)$ & $<0.001^{\text {* }}$ \\
\hline \multicolumn{4}{|l|}{ ADL } \\
\hline ADL [point, Median (Q1-Q3)] & 22.00 (20.00-30.00) & $32.50(23.00-42.25)$ & $<0.001^{\star \star}$ \\
\hline
\end{tabular}

Worse motor symptoms may aggravate patients' psychological and physical burden, which would increase the sense of guilt and despair. Besides, our study found that PIGD type was independently associated with PD-D. Accordingly, good management of motor symptoms is important for PD-D.
In our previous study (35), PIGD patients had more severe or faster neurodegeneration than TD group. In addition, PIGD severity might be related to the depletion of homovanillic acid, one of the metabolites of DA, in CSF (35). Moreover, in the current study, the decrease of DA might correlate with PD-D. 
TABLE 3 | Logistic regression analysis of factors associated with PD-D.

\begin{tabular}{lrccc}
\hline & B & OR & 95\% CI & $\boldsymbol{P}$ \\
\hline Age of onset & -0.033 & 0.967 & $0.935-1.000$ & 0.052 \\
UPDRS III score & 0.049 & 1.050 & $1.020-1.082$ & $0.001^{*}$ \\
PIGD type & -3.611 & 0.027 & $0.007-0.106$ & $<0.001^{\text {** }}$ \\
HAMA score & 0.285 & 1.330 & $1.216-1.454$ & $<0.001^{* *}$ \\
FS score & 0.111 & 1.117 & $1.005-1.241$ & $0.040^{\star}$ \\
RBDSQ score & 0.078 & 1.081 & $0.988-1.241$ & 0.090 \\
Constant number & -1.611 & 0.200 & & 0.152 \\
\hline
\end{tabular}

${ }^{*} P<0.05,{ }^{* *} P<0.01$. UPDRS III, Unified Parkinson's Disease Rating Scale III; PIGD, postural instability/gait difficulty; HAMA, the 14-item Hamilton Anxiety Scale; FS, the 14item Chalder Fatigue Scale; RBDSQ, the Rapid Eye Movement Sleep Behavior Disorder Screening Questionnaire; $B$, regression coefficient or intercept; OR, odds ratio; $\mathrm{Cl}$, confidence interval.

TABLE 4 | Levels of neurotransmitters in CSF from PD-D and PD-ND groups.

\begin{tabular}{lccc}
\hline & PD-ND group (24 cases) & PD-D group(52 cases) & $\boldsymbol{P}$ \\
\hline $\mathrm{DA}(\mathrm{fg} / \mathrm{mL}, \bar{X} \pm s)$ & $8.042 \pm 2.423$ & $6.025 \pm 2.214$ & $0.001^{*}$ \\
$5-\mathrm{HT}(\mathrm{fg} / \mathrm{mL}, \bar{X} \pm s)$ & $19.221 \pm 7.358$ & $14.796 \pm 8.365$ & 0.029 \\
$\mathrm{NE}(\mathrm{fg} / \mathrm{mL}, \bar{X} \pm s)$ & $483.004 \pm 140.534$ & $496.229 \pm 125.904$ & 0.683
\end{tabular}

${ }^{*} P<0.017$. DA, dopamine; 5-HT,5-hydroxytryptamine; NE, norepinephrine.

TABLE 5 | Correlation of HAMD scores with the levels of neurotransmitters in CSF from $P D$ patients.

\begin{tabular}{lcc}
\hline & $\boldsymbol{R}$ & $\boldsymbol{P}$ \\
\hline $\mathrm{DA}(\mathrm{fg} / \mathrm{ml})$ & -0.278 & $0.015^{*}$ \\
$5-\mathrm{HT}(\mathrm{fg} / \mathrm{ml})$ & -0.22 & 0.056 \\
$\mathrm{NE}(\mathrm{fg} / \mathrm{ml})$ & 0.056 & 0.63 \\
\hline
\end{tabular}

${ }^{*} P<0.017$. DA, dopamine; 5-HT, 5-hydroxytryptamine; NE, norepinephrine.

Therefore, the PIGD group might be vulnerable to PD-D. Much effort should be paid to PD patients with PIGD type to mitigate depression.

The comorbidity of depression and anxiety in PD patients was as high as $14-50 \%(36,37)$. In this study, the logistic regression analysis demonstrated that anxiety was one of the risk factors of PD-D. Anxiety and depression in PD patients often exist together, suggesting that they may have a common biochemical basis. They may both be related to the extensive serotonergic alteration and a more limited dopaminergic breakdown (38). Moreover, increased levels of inflammatory markers in CSF were strongly associated with depression, anxiety and other nonmotor symptoms of PD (39). Nonetheless, research suggests that the comorbidity of depression and anxiety might be resulted from the superposition of different pathophysiological mechanisms of anxiety and depression rather than the common mechanism (37).

Fatigue was previously considered as a manifestation of $\mathrm{PD}-\mathrm{D}$, but in recent years, it has been recognized as a nonmotor symptom independent of depression. A previous study showed that fatigue was correlated with depression (40). We
TABLE 6 | Logistic regression analysis of the relationship of DA in CSF and PD-D.

\begin{tabular}{lcccc}
\hline & B & OR & 95\% Cl & $P$ \\
\hline UPDRS III score & 0.03 & 1.031 & $0.949-1.112$ & 0.460 \\
PIGD type & -2.628 & 0.072 & $0.007-1.124$ & $0.042^{*}$ \\
HAMA score & 0.822 & 2.275 & $1.211-3.846$ & $0.008^{*}$ \\
DA & -1.012 & 0.364 & $0.172-0.816$ & $0.014^{*}$ \\
Constant number & 3.679 & 39.587 & & 0.154 \\
\hline
\end{tabular}

${ }^{*} P<0.05$. UPDRS III, Unified Parkinson's Disease Rating Scale III; PIGD, postural instability/gait difficulty; HAMA, the 14-item Hamilton Anxiety Scale; DA, dopamine; B, Regression coefficient or intercept; OR, odds ratio; $\mathrm{Cl}$, confidence interval.

further demonstrated that fatigue was one of the significant influencing factors of depression in PD patients. Our previous studies found that the decrease of serotonin dysfunction might be correlated with fatigue in PD patients (24). There was much interest in whether PD-D and fatigue shared the similar pathophysiologic mechanisms, for example, serotonergic dysfunction $(41,42)$. The underlying mechanism needs further investigation.

The research on the mechanisms of PD-D has not reached consensus. Studies showed that other chronic diseases, which also resulted in movement disorders, had lower incidences of depression than PD (28). What's more, depressive symptoms could appear before motor symptoms in PD patients (43). Therefore, as endogenous depression, PD-D is likely related to the characteristic pathology of PD. Theoretically, according to Braak stage of PD pathology (44), Lewy bodies deposit in the raphe nuclei, locus caeruleus, substantia nigra, and ventral tegmental area, and cause progressive loss of neurons and subsequent depletion of several neurotransmitters, including 5HT, NE, and DA, etc.

At Braak stage II, the serotonergic neurons are affected. A study reported that PD-D patients had reduced levels of 5-hydroxyindole acetic acid (5-HIAA, a metabolite of 5-HT) in CSF than PD-ND patients $(45,46)$. However, Olivola and investigators failed to find the changes of 5-HT in the CSF of PD-D patients (47). These studies, including our study, showed the lack of correlation between depression and 5-HT or 5HIAA in CSF. However, a positron emission tomography (PET) imaging study indicated a close correlation between PD-D and 5-HT transporter (48), although other similar studies failed to display consistent results $(49,50)$. Although there are many plausible theories explaining that deficits of the monoaminergic neurotransmitters are related to depression (51), the roles of serotonergic system on PD-D are less certain in human studies.

The level of DA is increasingly recognized as an important indicator for serious problems in PD-D; this is supported by pathological, experimental, and neuroimaging studies (52). The DA transporter availability was also proven to be reduced in PD-D in several PET studies $(12,53,54)$. As a DA receptor agonist, pramipexole (0.125-1.0 mg three times per day) could improve the depressive symptoms in PD patients, and $80 \%$ of the improvements was caused by a direct effect of treatment on depressive symptoms while $20 \%$ worked through the alleviation of motor symptoms (55). Pramipexole could upregulate the 
expression of dopamine receptor D3 (56). The same effect was found in Ropinirole (57) and a prospective multicenter study showed that Ropinirole (a median dose of $10 \mathrm{mg}$ ) could improve both anxious and depressive symptoms in PD patients (58). The anti-depressant effect also was obtained in Rasagiline with higher doses than those used for the control of motor symptoms (1 mg/day) (59) and it might be related with its role for dopamine-enhancing (60). Unfortunately, none of these studies reported an association between the levels of neurotransmitters in CSF and PD-D. The results of our study supported the model that PD-D arised as a result of the dysfunction in dopaminergic pathways (61). On the other hand, DA helped to improve the motor symptoms, which was an independent influencing factor for PD-D. In this study, it showed that in PDD patients, both the DA and 5-HT levels in CSF were decreased, however, only the level of DA in CSF was correlated with HAMD score. These results suggested that DA played a more significant role on PD-D. Therefore, it is plausible to speculate that dopaminergic-centered therapy may be much helpful for PD-D.

Most of NE neurons are distributed in the locus caeruleus. Previous investigations on the association between NE and PD-D were rather limited with relatively small sample sizes. Additionally, these studies chiefly focused on the damages of NE neurons and related innervations, and their correlation with PDD. For example, a study observed that neuronal loss in locus caeruleus was different between PD-D and PD-ND groups (62). Another PET study using ${ }^{11} \mathrm{C}$-RTI-32 as an in vivo marker for both DA and NE transporter binding, revealed that PD-D might be associated with the loss of NE innervations in the limbic system (12). Although the above studies showed clues implying the potential involvement of NE in PD-D, there was no direct evidence linking NE reduction in CSF to PD-D. In this study, the NE levels in CSF did not differ significantly between PD-D and PD-ND groups, and PD-D did not correlate with NE level in CSF. Therefore, NE might not be a critical neurotransmitter for PD-D.

This study has the following limitations. CSF samples were not obtained from all PD patients enrolled in this study due to difficulties including old age, hyperosteogeny, and intolerance of holding position for lumbar puncture, etc. CSF samples from PD patients with moderate and severe depression were also relatively limited. Thus, further investigations with more CSF samples, especially from moderate and severe $\mathrm{PD}-\mathrm{D}$ patients and prolonged follow-up time are much needed to support the results from the current study. What's more, as a retrospective and observational study, our study provided limited grounds for drawing definite conclusions. Longitudinal studies are required to elucidate how the depression in PD patients affects the progression and prognosis of these patients.

In summary, PD patients have a high frequency of mild and moderate depression. Motor symptoms, PIGD type, anxiety and fatigue are the significant influencing factors of $\mathrm{PD}-\mathrm{D}$. DA plays a more important role on PD-D. Results from this study provide new insights for the management of the above risk factors of PD-D and possible route for reduction of PD-D by targeting dopaminergic system.

\section{AUTHOR CONTRIBUTIONS}

T-HL drafting the manuscript, study design, analysis of data, accepts responsibility for conduct of research and will give final approval, acquisition of data, statistical analysis. PG study design, accepts responsibility for the conduct of research and will give final approval, acquisition of data. L-JZ, YH, S-YY, LL, ZJ, Q-JY, R-DW, L-XL, and Y-SP accepts responsibility for the conduct of research and will give final approval, acquisition of data. WZ study design, analysis of data, accepts responsibility for the conduct of research and will give final approval, acquisition of data, statistical analysis, study supervision.

\section{FUNDING}

This work was supported by The National Key Research and Development Program of China (2016YFC1306000, 2016YFC1306300), National Key R\&D Program of ChinaEuropean Commission Horizon 2020 (2017YFE0118800779238), The National Natural Science Foundation of China (81571229, 81071015, 30770745), The Key Project of National Natural Science Foundation of China (81030062), The Key Project of Natural Science Foundation of Beijing, China (B) (kz201610025030), The Key Project of Natural Science Foundation of Beijing, China (4161004, kz200910025001), The Natural Science Foundation of Beijing, China (7082032), National Key Basic Research Program of China (2011CB504100), Important National Science \& Technology Specific Projects (2011ZX09102-003-01), National Key Technology Research and Development Program of the Ministry of Science and Technology of China (2013BAI09B03), Project of Scientific and Technological Development of Traditional Chinese Medicine in Beijing (JJ2018-48), Project of Beijing Institute for Brain Disorders (BIBD-PXM2013_014226_07_000084), High Level Technical Personnel Training Project of Beijing Health System, China (2009-3-26), Project of Construction of Innovative Teams and Teacher Career Development for Universities and Colleges Under Beijing Municipality (IDHT20140514), Capital Clinical Characteristic Application Research (Z12110700100000, Z121107001012161), Beijing Healthcare Research Project, China (JING-15-2, JING-15-3), Excellent Personnel Training Project of Beijing, China (20071D0300400076), Natural Science Foundation of Capital Medical University (PYZ2018077), Basic-Clinical Research Cooperation Funding of Capital Medical University, China (2015-JL-PT-X04, 10JL49, 14JL15), Youth Research Funding, Beijing Tiantan Hospital, Capital Medical University, China (2014-YQN-YS-18, 2015-YQN-15, 2015-YQN-05, 2015-YQN-14, 2015-YQN-17). 


\section{REFERENCES}

1. Cosentino C, Nunez Y, Torres L. Frequency of non-motor symptoms in Peruvian patients with Parkinson's disease. Arq Neuropsiquiatr. (2013) 71:216-9. doi: 10.1590/0004-282X20130005

2. Goodarzi Z, Mrklas KJ, Roberts DJ, Jette N, Pringsheim T, Holroyd-Leduc J. Detecting depression in Parkinson disease: a systematic review and metaanalysis. Neurology (2016) 87:426-37. doi: 10.1212/wnl.0000000000002898

3. Becker C, Brobert GP, Johansson S, Jick SS, Meier CR. Risk of incident depression in patients with Parkinson disease in the UK. Eur J Neurol. (2011) 18:448-53. doi: 10.1111/j.1468-1331.2010.03176.x

4. Ziropadja L, Stefanova E, Petrovic M, Stojkovic T, Kostic VS. Apathy and depression in Parkinson's disease: the Belgrade PD study report. Parkinsonism Relat Disord. (2012) 18:339-42. doi: 10.1016/j.parkreldis.2011.11.020

5. Zhu J, Lu L, Pan Y, Shen B, Xu S, Hou Y, et al. Depression and associated factors in nondemented Chinese patients with Parkinson's disease. Clin Neurol Neurosurg. (2017) 163:142-8. doi: 10.1016/j.clineuro.2017.10.031

6. Quelhas R, Costa M. Anxiety, depression, and quality of life in Parkinson's disease. J Neuropsychiatry Clin Neurosci. (2009) 21:413-9. doi: 10.1176/appi.neuropsych.21.4.413

7. Leentjens AF, Moonen AJ, Dujardin K, Marsh L, Martinez-Martin P, Richard IH, et al. Modeling depression in Parkinson disease: diseasespecific and nonspecific risk factors. Neurology (2013) 81:1036-43. doi: 10.1212/WNL.0b013e3182a4a503

8. Ou R, Wei Q, Hou Y, Yuan X, Song W, Cao B, et al. Vascular risk factors and depression in Parkinson's disease. Eur J Neurol. (2018) 25:637-43. doi: 10.1111/ene.13551

9. Cui SS, Du JJ, Fu R, Lin YQ, Huang P, He YC, et al. Prevalence and risk factors for depression and anxiety in Chinese patients with Parkinson disease. BMC Geriatr. (2017) 17:270. doi: 10.1186/s12877-017-0666-2

10. Shin MS, Kim TW, Lee JM, Sung YH, Lim BV. Treadmill exercise alleviates depressive symptoms in rotenone-induced Parkinson disease rats. J Exerc Rehabil. (2017) 13:124-9. doi: 10.12965/jer.1734966.483

11. Gallagher DA, Schrag A. Psychosis, apathy, depression and anxiety in Parkinson's disease. Neurobiol Dis. (2012) 46:581-9. doi: 10.1016/j.nbd.2011.12.041

12. Remy P, Doder M, Lees A, Turjanski N, Brooks D. Depression in Parkinson's disease: loss of dopamine and noradrenaline innervation in the limbic system. Brain (2005) 128(Pt 6):1314-22. doi: 10.1093/brain/awh445

13. Williams JB. Standardizing the Hamilton Depression Rating Scale: past, present, and future. Eur Arch Psychiatry Clin Neurosci. (2001) 251(Suppl. 2):Ii6-12. doi: 10.1007/BF03035120

14. Hughes AJ, Daniel SE, Kilford L, Lees AJ. Accuracy of clinical diagnosis of idiopathic Parkinson's disease: a clinico-pathological study of 100 cases. J Neurol Neurosurg Psychiatry (1992) 55:181-4.

15. Tomlinson CL, Stowe R, Patel S, Rick C, Gray R, Clarke CE. Systematic review of levodopa dose equivalency reporting in Parkinson's disease. Mov Disord. (2010) 25:2649-53. doi: 10.1002/mds.23429

16. Starkstein S, Dragovic M, Jorge R, Brockman S, Merello M, Robinson RG, et al. Diagnostic criteria for depression in Parkinson's disease: a study of symptom patterns using latent class analysis. Mov Disord. (2011) 26:2239-45. doi: $10.1002 / \mathrm{mds} .23836$

17. Jankovic J, McDermott M, Carter J, Gauthier S, Goetz C, Golbe L, et al. Variable expression of Parkinson's disease: a base-line analysis of the DATATOP cohort. Parkinson Study Group Neurol. (1990) 40:1529-34.

18. Hoops S, Nazem S, Siderowf AD, Duda JE, Xie SX, Stern MB, et al. Validity of the MoCA and MMSE in the detection of MCI and dementia in Parkinson disease. Neurology (2009) 73:1738-45. doi: 10.1212/WNL.0b013e3181c34b47

19. Hamilton M. The assessment of anxiety states by rating. $\mathrm{Br} \mathrm{J} \mathrm{Med} \mathrm{Psychol.}$ (1959) 32:50-5.

20. Chalder T, Berelowitz G, Pawlikowska T, Watts L, Wessely S, Wright D, et al. Development of a fatigue scale. J Psychosom Res. (1993) 37:147-53.

21. Zea-Sevilla MA, Martinez-Martin P. Rating scales and questionnaires for assessment of sleep disorders in Parkinson's disease: what they inform about? J Neural Transm. (2014) 121(Suppl. 1):S33-40. doi: 10.1007/s00702-014-1217-z

22. Visser M, Marinus J, Stiggelbout AM, Van Hilten JJ. Assessment of autonomic dysfunction in Parkinson's disease: the SCOPA-AUT. Mov Disord. (2004) 19:1306-12. doi: 10.1002/mds.20153
23. Walters AS, LeBrocq C, Dhar A, Hening W, Rosen R, Allen RP, et al. Validation of the international restless legs syndrome study group rating scale for restless legs syndrome. Sleep Med. (2003) 4:121-32. doi: 10.1016/S1389-9457(02)00258-7

24. Zuo LJ, Yu SY, Hu Y, Wang F, Piao YS, Lian TH, et al. Serotonergic dysfunctions and abnormal iron metabolism: relevant to mental fatigue of Parkinson disease. Sci Rep. (2016) 6:19. doi: 10.1038/s41598-0160018-z

25. Gallagher DA, Lees AJ, Schrag A. What are the most important nonmotor symptoms in patients with Parkinson's disease and are we missing them? Mov Disord. (2010) 25:2493-500. doi: 10.1002/mds.23394

26. Muller B, Assmus J, Herlofson K, Larsen JP, Tysnes OB. Importance of motor vs. non-motor symptoms for health-related quality of life in early Parkinson's disease. Parkinsonism Relat Disord. (2013) 19:1027-32. doi: 10.1016/j.parkreldis.2013.07.010

27. Carod-Artal FJ, Ziomkowski S, Mourao Mesquita H, Martinez-Martin P. Anxiety and depression: main determinants of health-related quality of life in Brazilian patients with Parkinson's disease. Parkinsonism Relat Disord. (2008) 14:102-8. doi: 10.1016/j.parkreldis.2007.06.011

28. Sagna A, Gallo JJ, Pontone GM. Systematic review of factors associated with depression and anxiety disorders among older adults with Parkinson's disease. Parkinsonism Relat Disord. (2014) 20:708-15. doi: 10.1016/j.parkreldis.2014.03.020

29. Reijnders JS, Ehrt U, Weber WE, Aarsland D, Leentjens AF. A systematic review of prevalence studies of depression in Parkinson's disease. Mov Disord. (2008) 23:183-9; quiz: 313. doi: 10.1002/mds.21803

30. Baxter AJ, Charlson FJ, Cheng HG, Shidhaye R, Ferrari AJ, Whiteford HA. Prevalence of mental, neurological, and substance use disorders in China and India: a systematic analysis. Lancet Psychiatry (2016) 3:832-41. doi: 10.1016/s2215-0366(16)30139-0

31. Aarsland D, Pahlhagen S, Ballard CG, Ehrt U, Svenningsson P. Depression in Parkinson disease-epidemiology, mechanisms and management. Nat Rev Neurol. (2011) 8:35-47. doi: 10.1038/nrneurol.2011.189

32. Ravina B, Elm J, Camicioli R, Como PG, Marsh L, Jankovic J, et al. The course of depressive symptoms in early Parkinson's disease. Mov Disord. (2009) 24:1306-11. doi: 10.1002/mds.22572

33. Even C, Weintraub D. Is depression in Parkinson's disease (PD) a specific entity? J Affect Disord. (2012) 139:103-12. doi: 10.1016/j.jad.2011.07.002

34. Schrag A, Jahanshahi M, Quinn NP. What contributes to depression in Parkinson's disease? Psychol Med. (2001) 31:65-73. doi: 10.1017/s0033291799003141

35. Zuo LJ, Piao YS, Li LX, Yu SY, Guo P, Hu Y, et al. Phenotype of postural instability/gait difficulty in Parkinson disease: relevance to cognitive impairment and mechanism relating pathological proteins and neurotransmitters. Sci Rep. (2017) 7:44872. doi: 10.1038/srep44872

36. Dissanayaka NN, Sellbach A, Matheson S, O’Sullivan JD, Silburn PA, Byrne GJ, et al. Anxiety disorders in Parkinson's disease: prevalence and risk factors. Mov Disord. (2010) 25:838-45. doi: 10.1002/mds.22833

37. Negre-Pages L, Grandjean H, Lapeyre-Mestre M, Montastruc JL, Fourrier A, Lepine JP, et al. Anxious and depressive symptoms in Parkinson's disease: the French cross-sectionnal DoPaMiP study. Mov Disord. (2010) 25:157-66. doi: $10.1002 / \mathrm{mds} .22760$

38. Maillet A, Krack P, Lhommee E, Metereau E, Klinger H, Favre E, et al. The prominent role of serotonergic degeneration in apathy, anxiety and depression in de novo Parkinson's disease. Brain (2016) 139(Pt 9):2486-502. doi: 10.1093/brain/aww162

39. Lindqvist D, Hall S, Surova Y, Nielsen HM, Janelidze S, Brundin L, et al. Cerebrospinal fluid inflammatory markers in Parkinson's disease-associations with depression, fatigue, and cognitive impairment. Brain Behav Immun. (2013) 33:183-9. doi: 10.1016/j.bbi.2013.07.007

40. Stocchi F, Abbruzzese G, Ceravolo R, Cortelli P, D’Amelio M, De Pandis MF, et al. Prevalence of fatigue in Parkinson disease and its clinical correlates. Neurology (2014) 83:215-20. doi: 10.1212/wnl.0000000000000587

41. Pavese N, Metta V, Bose SK, Chaudhuri KR, Brooks DJ. Fatigue in Parkinson's disease is linked to striatal and limbic serotonergic dysfunction. Brain (2010) 133:3434-43. doi: 10.1093/brain/awq268

42. Politis M, Niccolini F. Serotonin in Parkinson's disease. Behav Brain Res. (2015) 277:136-45. doi: 10.1016/j.bbr.2014.07.037 
43. Fang F, Xu Q, Park Y, Huang X, Hollenbeck A, Blair A, et al. Depression and the subsequent risk of Parkinson's disease in the NIH-AARP Diet and Health Study. Mov Disord. (2010) 25:1157-62. doi: 10.1002/mds.23092

44. Lees AJ, Hardy J, Revesz T. Parkinson's disease. Lancet (2009) 373:2055-66. doi: 10.1016/s0140-6736(09)60492-x

45. Kostic VS, Djuricic BM, Covickovic-Sternic N, Bumbasirevic L, Nikolic M, Mrsulja BB. Depression and Parkinson's disease: possible role of serotonergic mechanisms. J Neurol. (1987) 234:94-6.

46. Mayeux R, Stern Y, Cote L, Williams JB. Altered serotonin metabolism in depressed patients with parkinson's disease. Neurology (1984) 34:642-6.

47. Olivola E, Pierantozzi M, Imbriani P, Liguori C, Stampanoni Bassi M, Conti M, et al. Serotonin impairment in CSF of PD patients, without an apparent clinical counterpart. PLoS ONE (2014) 9:e101763. doi: 10.1371/journal.pone.0101763

48. Boileau I, Warsh JJ, Guttman M, Saint-Cyr JA, McCluskey T, Rusjan P, et al. Elevated serotonin transporter binding in depressed patients with Parkinson's disease: a preliminary PET study with [11C]DASB. Mov Disord. (2008) 23:1776-80. doi: 10.1002/mds. 22212

49. Politis M, Wu K, Loane C, Turkheimer FE, Molloy S, Brooks DJ, et al. Depressive symptoms in PD correlate with higher 5-HTT binding in raphe and limbic structures. Neurology (2010) 75:1920-7. doi: 10.1212/WNL.0b013e3181feb2ab

50. Strecker K, Wegner F, Hesse S, Becker GA, Patt M, Meyer PM, et al. Preserved serotonin transporter binding in de novo Parkinson's disease: negative correlation with the dopamine transporter. J Neurol. (2011) 258:1926. doi: 10.1007/s00415-010-5666-5

51. Schildkraut JJ. The catecholamine hypothesis of affective disorders: a review of supporting evidence. 1965. J Neuropsychiatry Clin Neurosci. (1995) 7:524-33; discussion 523-4. doi: 10.1176/jnp.7.4.524

52. Schapira AHV, Chaudhuri KR, Jenner P. Non-motor features of Parkinson disease. Nat Rev Neurosci. (2017) 18:435-50. doi: 10.1038/nrn.2017.62

53. Vriend C, Raijmakers P, Veltman DJ, van Dijk KD, van der Werf YD, Foncke EM, et al. Depressive symptoms in Parkinson's disease are related to reduced [123I]FP-CIT binding in the caudate nucleus. J Neurol Neurosurg Psychiatry (2014) 85:159-64. doi: 10.1136/jnnp-2012-304811

54. Vuckovic MG, Wood RI, Holschneider DP, Abernathy A, Togasaki DM, Smith A, et al. Memory, mood, dopamine, and serotonin in the 1-methyl4-phenyl-1,2,3,6-tetrahydropyridine-lesioned mouse model of basal ganglia injury. Neurobiol Dis. (2008) 32:319-27. doi: 10.1016/j.nbd.2008.07.015

55. Barone P, Poewe W, Albrecht S, Debieuvre C, Massey D, Rascol O, et al. Pramipexole for the treatment of depressive symptoms in patients with
Parkinson's disease: a randomised, double-blind, placebo-controlled trial. Lancet Neurol. (2010) 9:573-80. doi: 10.1016/s1474-4422(10)70106-x

56. Tokunaga N, Choudhury ME, Nishikawa N, Nagai M, Tujii T, Iwaki H, et al. Pramipexole upregulates dopamine receptor $\mathrm{D}(2)$ and $\mathrm{D}(3)$ expression in rat striatum. J Pharmacol Sci. (2012) 120:133-7. doi: 10.1254/jphs. 12096SC

57. Kang SY, Ryu HS, Sunwoo MK, Kim SJ, Baik JS, Park MY, et al. Sleepiness and depression in Parkinson's Disease patients treated with ropinirole and levodopa. J Mov Disord. (2017) 10:123-9. doi: 10.14802/jmd.17048

58. Rektorova I, Balaz M, Svatova J, Zarubova K, Honig I, Dostal V, et al. Effects of ropinirole on nonmotor symptoms of Parkinson disease: a prospective multicenter study. Clin Neuropharmacol. (2008) 31:261-6. doi: 10.1097/WNF.0b013e31815d25ce

59. Korchounov A, Winter Y, Rossy W. Combined beneficial effect of rasagiline on motor function and depression in de novo PD. Clin Neuropharmacol. (2012) 35:121-4. doi: 10.1097/WNF.0b013e31823b1da8

60. Smith KM, Eyal E, Weintraub D. Combined rasagiline and antidepressant use in Parkinson disease in the ADAGIO study: effects on nonmotor symptoms and tolerability. JAMA Neurol. (2015) 72:88-95. doi: 10.1001/jamaneurol.2014.2472

61. Dunlop BW, Nemeroff CB. The role of dopamine in the pathophysiology of depression. Arch Gen Psychiatry (2007) 64:327-37. doi: 10.1001/archpsyc.64.3.327

62. Frisina PG, Haroutunian V, Libow LS. The neuropathological basis for depression in Parkinson's disease. Parkinson Relat Disord. (2009) 15:144-8. doi: 10.1016/j.parkreldis.2008.04.038

Conflict of Interest Statement: The authors declare that the research was conducted in the absence of any commercial or financial relationships that could be construed as a potential conflict of interest.

The reviewer FD and handling editor declared their shared affiliation at time of review.

Copyright (c) 2019 Lian, Guo, Zuo, Hu, Yu, Liu, Jin, Yu, Wang, Li, Piao and Zhang. This is an open-access article distributed under the terms of the Creative Commons Attribution License (CC BY). The use, distribution or reproduction in other forums is permitted, provided the original author(s) and the copyright owner(s) are credited and that the original publication in this journal is cited, in accordance with accepted academic practice. No use, distribution or reproduction is permitted which does not comply with these terms. 